\title{
Diminutive Digits Discern Delicate Details: Fingertip Size and the Sex Difference in Tactile Spatial Acuity
}

\author{
Ryan M. Peters, ${ }^{1}$ Erik Hackeman, ${ }^{2}$ and Daniel Goldreich ${ }^{1,2}$ \\ ${ }^{1}$ Department of Psychology, Neuroscience \& Behaviour, McMaster University, Hamilton, Ontario L8S 4K1, Canada, and ${ }^{2}$ Department of Occupational \\ Therapy, Duquesne University, Pittsburgh, Pennsylvania 15282
}

We have observed that passive tactile spatial acuity, the ability to resolve the spatial structure of surfaces pressed upon the skin, differs subtly but consistently between the sexes, with women able to perceive finer surface detail than men. Eschewing complex central explanations, we hypothesized that this sex difference in somatosensory perception might result from simple physical differences between the fingers of women and men. To investigate, we tested 50 women and 50 men on a tactile grating orientation task and measured the surface area of the participants' index fingertips. In subsets of participants, we additionally measured finger skin compliance and optically imaged the fingerprint microstructure to count sweat pores. We show here that tactile perception improves with decreasing finger size, and that this correlation fully explains the better perception of women, who on average have smaller fingers than men. Indeed, when sex and finger size are both considered in statistical analyses, only finger size predicts tactile acuity. Thus, a man and a woman with fingers of equal size will, on average, enjoy equal tactile acuity. We further show that sweat pores, and presumably the Merkel receptors beneath them, are packed more densely in smaller fingers.

\section{Introduction}

The ability to resolve patterned surfaces pressed upon the stationary fingertip differs between the sexes, according to recent studies, with women able to perceive finer surface detail than men (Van Boven et al., 2000; Goldreich and Kanics, 2003, 2006). For instance, Goldreich and Kanics (2003) tested 43 blind and 47 sighted participants on a tactile grating orientation task. The blind participants significantly outperformed their sighted peers, and within both the blind and sighted groups, women significantly outperformed men. A recently concluded grating orientation study with a new group of 28 blind and 57 sighted participants has again shown this result (M. Wong, V. Gnanakumaran, D. Goldreich, unpublished observations).

Why do women outperform men on tests of tactile spatial acuity? Eschewing more complex explanations, we hypothesized that the superior tactile perception of women might result from physical differences between the fingers of women and men. We considered two hypotheses: (1) If women's fingers are more compliant than those of men (Woodward, 1993), a force-controlled stimulus would indent more deeply into women's fingers, perhaps resulting in superior perception, and (2) as women's fingers are smaller than men's (Dillon et al., 2001), Merkel receptor den-

Received July 29, 2009; revised Sept. 17, 2009; accepted 0ct. 22, 2009.

This research was supported by grants to D.G. from the National Eye Institute (US) and the Natural Sciences and Engineering Research Council (Canada). We thank Caitlin Hurd and John Mclntyre for the finger area and sweat pore measurements, and Raagula Sivayoganathan for help with experiments. We thank Deda Gillespie for assistance with equipment design and construction, and for many fruitful conversations. We thank www.myctrring.com for permission to use the hand drawing in Figure $1 a$.

Correspondence should be addressed to Daniel Goldreich, Department of Psychology, Neuroscience \& Behaviour, McMaster University, Hamilton, 0N L8S 4K1, Canada. E-mail: goldrd@mcmaster.ca.

DOI:10.1523/JNEUROSCI.3684-09.2009

Copyright $\odot 2009$ Society for Neuroscience $\quad$ 0270-6474/09/2915756-06\$15.00/0 sity might be higher in women's fingers, again resulting in superior perception.

The following reasoning led us to the second hypothesis: Merkel cells, activated by static skin displacement, are thought to mediate tactile spatial perception (Iggo and Muir, 1969; Ogawa, 1996; Johnson, 2001). These receptors are difficult to visualize anatomically (Boulais and Misery, 2007), and their density with respect to finger size is unknown. However, Meissner corpuscles, activated by low-frequency skin vibration and easily visualized anatomically, are more densely distributed in smaller fingers (Bolton et al., 1966; Dillon et al., 2001; Nolano et al., 2003). Indeed, homologous fingers in different individuals probably have the same number of Meissner corpuscles (Bolton et al., 1966; Dillon et al., 2001). If Merkel cells, like Meissner corpuscles, are more densely packed in smaller fingers, then presumably the fingers of women would be endowed with greater spatial resolving power than those of men.

Here we show that tactile perception indeed correlates with finger size, and that this effect fully explains the superior tactile spatial acuity of women compared to men.

\section{Materials and Methods}

Participants. One hundred undergraduate participants (50 women, 50 men; $18-27$ years old), were recruited from Duquesne University ( $n=$ $48)$ and McMaster University $(n=52)$. The study protocol was approved by the review boards of both universities. Entrance criteria ensured that participants had no index finger cuts, scars, or calluses, and that they were (by self-report) free from nervous system disorders, dyslexia, and diabetes. Two participants (one male, one female) were unable to complete the majority of testing blocks, so their data were omitted from analysis. The finger scan from a third participant (female) was inadvertently overwritten. Thus, the analyses reported here derive from 97 participants (Duquesne: 23 women, 23 men; McMaster: 25 women, 26 men). The age 

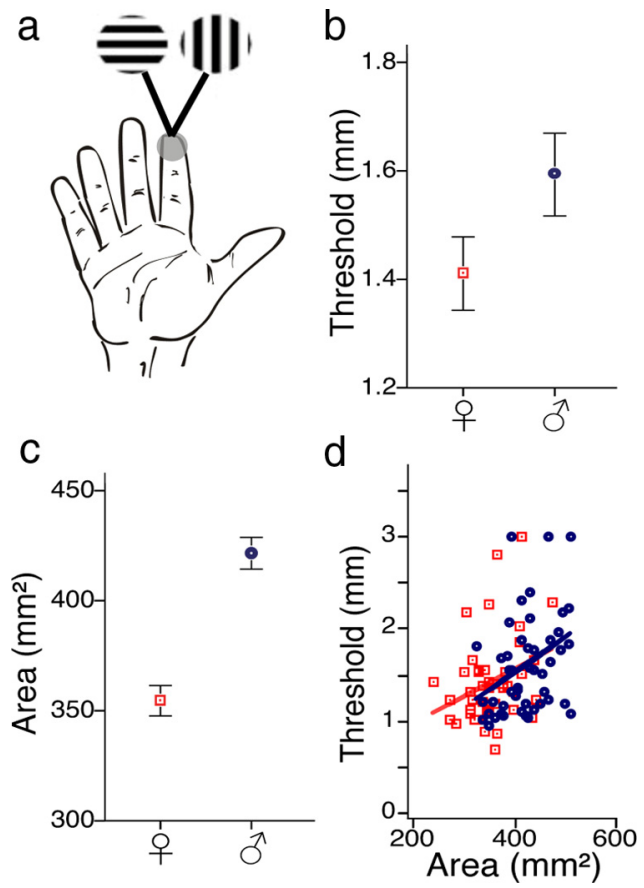

Figure 1. Perceptual data and finger size. $\boldsymbol{a}$, Two-interval forced-choice GOT. An adaptive procedure estimated the width of grooves whose orientation the participant could distinguish with 76\% probability (GOT threshold). Hand drawing retrieved from www.myctrring.com with permission. $\boldsymbol{b}, \mathrm{GOT}$ thresholds by sex (means $\pm 1 \mathrm{SE}$ ). Lower thresholds correspond to better acuity. $\boldsymbol{c}$, Index finger distal phalanx surface area by sex (means \pm 1 SE). $\boldsymbol{d}$, Scatterplot of threshold versus distal phalanx surface area, with female (red) and male (blue) regression lines. Women: red $\square$; men: blue $\bigcirc$.

distributions of these women (mean 20.5 years, SD 1.5 years) and men (mean 20.4 years, SD 1.2 years) were well matched. The young-adult age range of the participants protected the tactile acuity data against variance caused by the detrimental effects of aging (Stevens and Choo, 1996; Goldreich and Kanics, 2003, 2006).

Sensory testing. We used a two-interval forced-choice (2-IFC) grating orientation task (GOT) to assess passive (finger stationary) tactile spatial acuity. The GOT has several advantages over the two-point (calipers) test as a measure of spatial acuity (Johnson and Phillips, 1981; Craig and Johnson, 2000). The testing apparatus and procedure are described in detail in Goldreich et al. (2009). Briefly, grooved surfaces (20 squarewave gratings with groove widths ranging from 0.25 to $3.10 \mathrm{~mm}$, in 0.15 $\mathrm{mm}$ increments) rose under computer control to contact the distal pad of the dominant index finger $(4 \mathrm{~cm} / \mathrm{s}$ onset velocity, $1 \mathrm{~s}$ duration, $50 \mathrm{~g}$ force). In each trial, the finger was contacted twice, with surfaces of identical groove width but orthogonal orientations (perpendicular or parallel to the long axis of the finger, with the order of presentation chosen randomly) (Fig. $1 a)$. The participant indicated whether the perpendicular orientation occurred in the first or second interval. Threshold was defined as the width of the grooves whose orientation the participant could discern with $76 \%$ probability, corresponding to d-prime $=1$ on this 2 -IFC task (Gescheider, 1997).

To assess threshold efficiently, we used adaptive procedures, selecting the groove width on each trial according to the participants' previous responses. In the Duquesne experiments, we used a two-down one-up staircase, with 14 reversals per testing block, as described by Goldreich and Kanics (2003). The Duquesne data consisted of two testing blocks per participant, taken from a larger experiment of eight blocks (the two blocks were collected in a lit room with the participant's eyes open, corresponding to the McMaster testing condition; the other blocks, not relevant to the current study, tested different lighting conditions). In the McMaster experiments, we used an adaptive Bayesian procedure, modified from Kontsevich and Tyler (1999) (see supplemental Material, available at www.jneurosci.org). The McMaster data consisted of eight testing blocks of 40 trials each. Although the Duquesne data were collected by staircase, they were subsequently analyzed with the Bayesian method, so that both data sets yielded the same ( $76 \%$ correct) threshold measure.

Finger measurements. We determined hand dominance by survey (modified from Oldfield, 1971), then scanned the distal index finger of the dominant hand at 300 dpi (Epson Perfection 1260 scanner, Epson Electronics America) for subsequent finger measurements (NIH image 1.63 and Image $1.40 ; \mathrm{NIH}$, Bethesda, MD). All finger area and sweat pore measurements were made independently by two observers (not authors) who were blind to the participants' sex and tactile thresholds.

To measure fingertip surface area, we traced the image of each finger from the distal interphalangeal crease to the end of the finger (we refer to this entire distal phalanx area as the "fingertip"). In case of multiple distal interphalangeal creases, we traced the most prominent crease; when two creases were equally prominent, we drew a line parallel to the two creases and equidistant from them (see Fig. $3 a$ ). Fingertip surface area was measured for every participant. To measure sweat pore density, we recalled 15 participants from the McMaster pool, spanning the ranges of fingertip sizes observed in the study, and coated each participant's distal index finger pad with water-soluble finger paint (Crayola), which settles into the pores, then scanned at high resolution ( $2400 \mathrm{dpi})$. Because the spacing between sweat pores within a fingerprint ridge differed from the spacing between pores on adjacent ridges, we separately measured 20 within-ridge and 20 between-ridge pore pair distances per participant, and derived pore density (pores $/ \mathrm{mm}^{2}$ ) from the participant's average within-ridge $(\mathrm{w})$ and between-ridge (b) pore-to-pore spacing $(\mathrm{mm})$ : density $=1 /(\mathrm{wb})$.

We assessed interobserver reliability in two ways: (1) by Pearson's $r$, and (2) by calculating, for each participant, the absolute value of the observer 1-observer 2 difference score, divided by the mean of the observer 1 and observer 2 scores. Interobserver agreement was excellent: $r=0.997$, two-tailed $p<0.001$ (finger area), $r=0.882$, two-tailed $p<$ 0.001 (sweat pore spacing); average difference score measure $=1 \%$ (finger area) and $6 \%$ (sweat pore spacing). For each participant, we averaged the two observers' measurements for subsequent analysis.

At Duquesne University, we measured skin compliance as the skin indentation depth produced by a 0.5 -inch-diameter surface at $50 \mathrm{~g}$ contact force (the same stimulus area and force used in our grating orientation task). We built a laser-based measurement system for this purpose (supplemental Fig. 1, available at www.jneurosci.org as supplemental material). Skin compliance measurements taken on the Duquesne participants showed no significant difference between men and women, nor correlated with GOT threshold. In contrast, fingertip area measurements taken on the same participants did differ significantly by sex and correlate with GOT threshold. Therefore, in our subsequent (McMaster University) experiments, we measured fingertip area only.

Statistical analysis. We performed conventional statistical tests ( $t$ tests, correlations, ANCOVA, regression) with SPSS 16, using a significance criterion of 0.05 . We report one-tailed $p$ values for all tests, as each of our alternative hypotheses predicted a specific effect direction. We programmed Bayesian analysis (Sivia and Skilling, 2006) in LabVIEW 7.0 (National Instruments). The Bayes factor, $B_{12}$, comparing models $M_{1}$ and $\mathrm{M}_{2}$, is a likelihood ratio: $B_{12}=P\left(\mathrm{D} \mid \mathrm{M}_{1}\right) / P\left(\mathrm{D} \mid \mathrm{M}_{2}\right)$, where $P(\mathrm{D} \mid \mathrm{M})$ is the probability of the data, D, given model M (Kass and Raftery, 1995, Goodman, 1999). For example, a Bayes factor of 10 means that Model 1 predicted the data with 10 times the probability of Model 2. See supplemental Material, available at www.jneurosci.org, for details of the Bayesian method.

\section{Results}

We used an automated system (Goldreich et al., 2009) to assess the passive tactile spatial acuity of women and men on a grating orientation task (Fig. 1a). The results confirmed the sex difference in tactile acuity (Fig. $1 b$; unpaired $t=1.79, p=0.038$ ). In fact, the mean performance difference between the sexes, 0.18 $\mathrm{mm}$, was identical to that reported previously (Goldreich and 
Kanics, 2003). We next investigated the cause of this tactile sex difference.

According to Hypothesis 1, women outperform men because women have more compliant fingers. The data refuted this hypothesis, as we found that skin compliance was neither greater in women (unpaired $t$ test, $p=0.9$ ) nor predicted tactile acuity (GOT threshold vs compliance correlation, $p=0.8$ ). In fact, the data showed a (nonsignificant) trend of greater compliance in male than in female fingers, opposite the direction predicted by $\mathrm{Hy}-$ pothesis 1 (supplemental Fig. 2, available at www.jneurosci.org as supplemental material).

According to Hypothesis 2, women outperform men because women have smaller fingers. As expected, women's fingers were significantly smaller than men's (Fig. 1c) (unpaired $t=6.72, p<0.001$ ). To investigate whether this accounted for the better acuity of the women, we performed a sex-by-fingertip-area ANCOVA on the acuity data. Strikingly, and as predicted by Hypothesis 2, this analysis revealed a highly significant main effect of fingertip area $\left(F_{(1,94)}=11.7, p<0.001\right)$ but no effect of $\operatorname{sex}\left(F_{(1,94)}=0.148, p=\right.$ $0.65)$. Thus, when finger size was considered, the apparent sex effect on acuity vanished. Indeed, tactile thresholds correlated with finger area not only across the entire participant sample (Pearson's $r=0.37, p<0.001$ ) but also within both the male $(r=0.36, p=0.005)$ and female $(r=0.30, p=0.021)$ groups (Fig. $1 d$ ). These results strongly support Hypothesis 2: tactile spatial acuity is determined not by sex per se but by finger size.

We independently verified this conclusion by using robust Bayesian analysis to compare four competing models that represented different assumptions about the source(s) of variance in the participants' data (Fig. 2). A null model interpreted participants' thresholds as random samples drawn from a single Gaussian population distribution; a sex model specified separate male and female distributions; a size model interpreted thresholds as a linear function of fingertip area; and a size-and-sex model specified offset male and female functions of fingertip area. The size model emerged the clear winner (size model likelihood: 171, 76, and 6.5 relative to null, sex, and size-and-sex models), supporting the conclusion that finger size, not sex, determines tactile spatial acuity. This model revealed that tactile thresholds increase at a rate of $0.25 \mathrm{~mm}$ per $\mathrm{cm}^{2}$ fingertip surface area ( $95 \%$ confidence interval: $0.11-0.40 \mathrm{~mm} / \mathrm{cm}^{2}$ ).

The finding that smaller fingers have better acuity suggests an inverse relationship between finger size and the density of Merkel cells, the putative mechanotransducers for statically impressed stimuli (Iggo and Muir, 1969; Ogawa, 1996; Johnson, 2001). Given that Merkel cells cluster around the bases of sweat pores in the deep epidermis (Yamada et al., 1996), we reasoned that sweat pore density would provide a measurable correlate of Merkel cluster density. High-resolution scans revealed that sweat pore density is indeed greater in smaller fingers (Fig. 3) (sweat pore density vs fingertip area, Pearson's $r=-0.50, p=0.028, n=15)$, suggesting that Merkel cells pack more densely in smaller fingers.
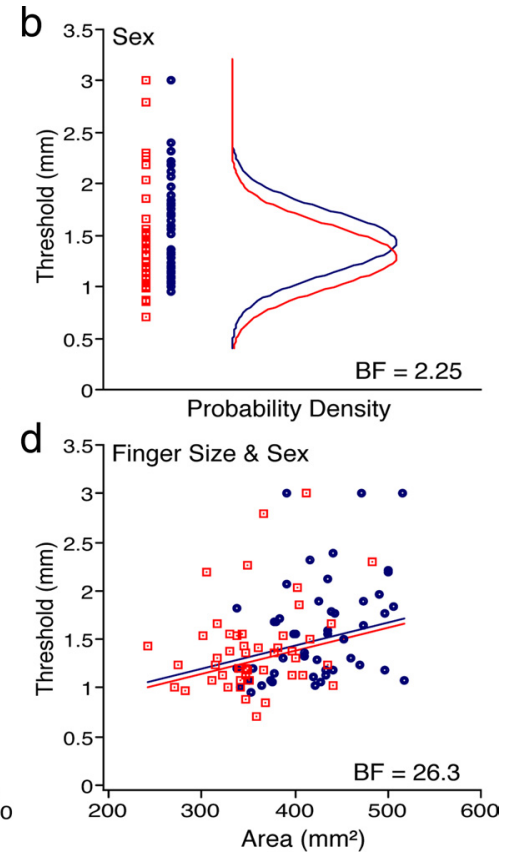

(2)

est-fit curves are shown for four models. The data points (participants' GOT thresholds) are identical in the four plots; the models differ in how they consider the data to have been generated. $\boldsymbol{a}$, Null model: the data derive from a single Gaussian population distribution. $\boldsymbol{b}$, Sex model: female and male data (left-right offset for clarity) originate from separate (red and blue) Gaussian populations. $c$, Finger size model: the data derive from a linear trend on fingertip area.d, Finger-size-and-sex model. Women: red $\square$; men: blue $\bigcirc$. Bayes factors (BF) are likelihoods relative to the null model.

\section{Discussion}

We have confirmed that women possess on average finer passive tactile spatial acuity than men, and we have discovered a surprisingly simple explanation for this: tactile spatial perception improves with decreasing finger size. Indeed, we find that when sex and finger size are both considered in statistical analyses, only finger size predicts tactile acuity. Thus, a man and a woman with fingers of equal size will, on average, experience equal tactile acuity.

Why does finger size affect spatial acuity? The high density of Meissner corpuscles in small fingers (Bolton et al., 1966; Dillon et al., 2001; Nolano et al., 2003) presumably does not improve spatial acuity, because Meissner corpuscles activate rapidly adapting type-I (RA1) afferents that interfere with fine spatial perception (Bensmaïa et al., 2006). In contrast, a high density of Merkel cells could improve spatial acuity.

When a structured surface presses against the skin, it evokes a spatially modulated discharge pattern in the underlying slowly adapting type-I (SA1) afferent axon population (Phillips and Johnson, 1981a). The precision of this neural image depends crucially on SA1 innervation density and receptive field size. The fingertip, for instance, has greater SA1 density (Johansson and Vallbo, 1979), smaller SA1 receptive fields (Schady and Torebjörk, 1983), and correspondingly better spatial acuity than does the finger base (Gibson and Craig, 2002). Anatomical (Güçlü et al., 2008) and physiological (Johansson, 1978) evidence suggests that SA1s branch to innervate several clusters of Merkel cells (Fig. 4a).

We suggest that finger size affects acuity because Merkel cells, like Meissner corpuscles, are distributed more densely in smaller fingers; thus, smaller fingers produce a finer-grained afferent neural image of an impressed tactile stimulus. This interpretation is based on two key assumptions, to be investigated by future 

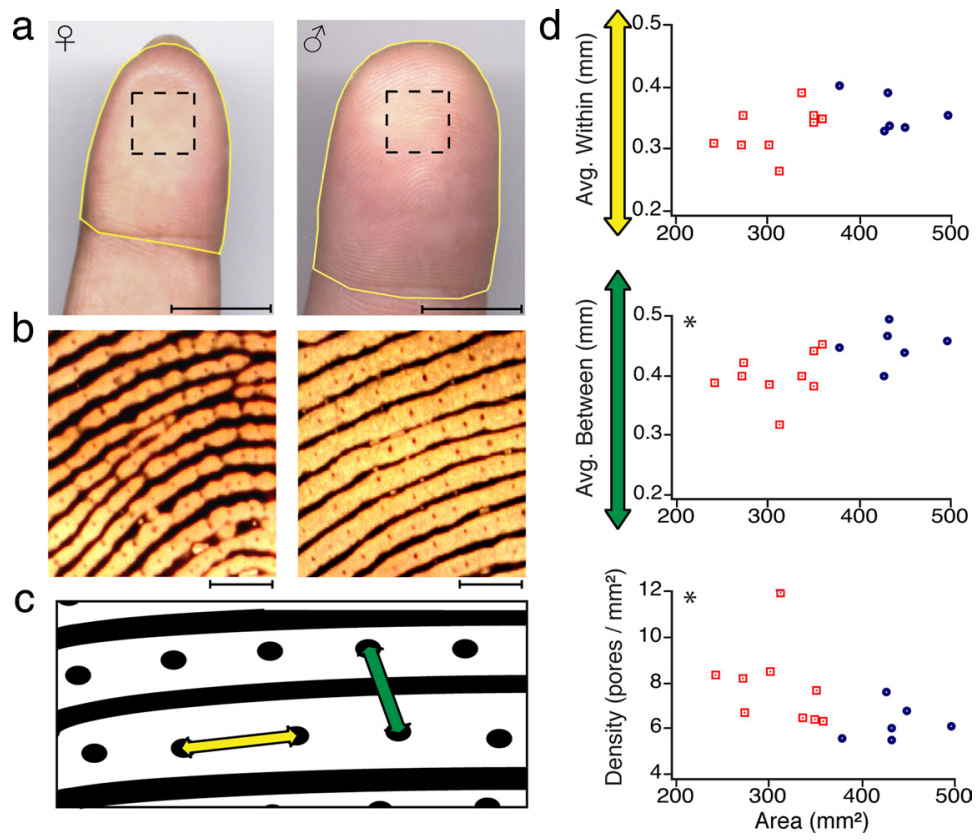

Figure 3. Finger surface microstructure. $\boldsymbol{a}$, Scans from index fingers of a woman (left) and man (right) traced (yellow) for area measurement (scale bar, $1 \mathrm{~cm}$ ). $\boldsymbol{b}$, Portions of $2400 \mathrm{dpi}$ scans taken from boxed regions in $\boldsymbol{a}$ after staining (scale bar, $1 \mathrm{~mm}$ ). Sweat pores (punctate stain) are more densely distributed in the smaller finger. $c$, Within-ridge (yellow arrow) and between-ridge (green arrow) pore-to-pore measurements were taken from 15 participants. Dots: sweat pores; lines: finger print grooves. $\boldsymbol{d}$, Pore-to-pore within-ridge distance (top), between-ridge distance (middle), and sweat pore density (lower) versus fingertip surface area. Women: red $\square$; men: blue $\bigcirc$. ${ }^{*}$ correlation $p<0.05$.
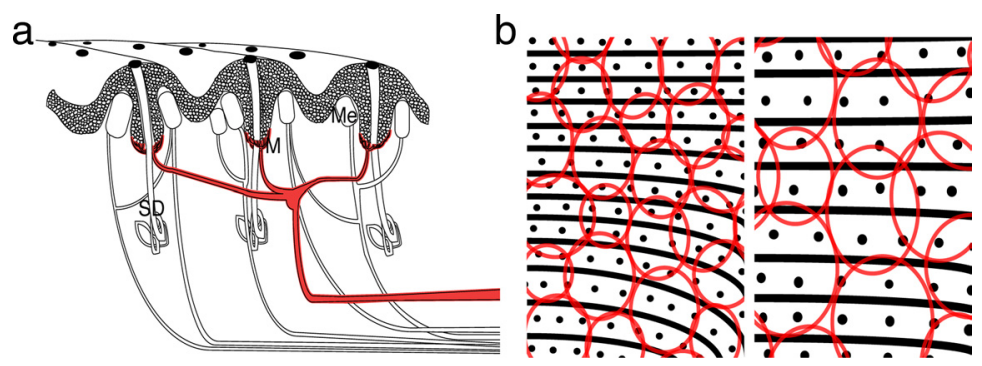

Figure 4. Proposed receptor anatomy and effect of finger size. $\boldsymbol{a}$, Schematic cross-section through finger. An SA1 axon (red) branches to Merkel cell clusters (M) encircling sweat ducts (SD) beneath papillary ridges (stippled). RA1 axons (uncolored) innervate Meissner corpuscles (Me). $\boldsymbol{b}$, We propose that SA1 receptive fields (ellipses) are more densely packed in smaller fingers (left).

lations between these factors and finger size, but such correlations are plausible. The stratum corneum, the skin's outermost layer, is slightly thicker in male than in female fingers (Fruhstorfer et al., 2000). A positive correlation between stratum corneum thickness and finger size could account in part for the effect of finger size on acuity. Skin temperature and hydration are also greater in male than in female fingers (Verrillo et al., 1998; see also Lévêque et al., 2000). Skin temperature might plausibly increase with finger size, as larger fingers have smaller surface-tovolume ratios, so should undergo slower heat loss. The effects of small variations in skin temperature and endogenous skin hydration on tactile spatial acuity are unclear, however.

Our statistical analyses indicate that when finger size is considered there is no longer a detectable independent effect of sex on tactile spatial acuity; that is, the sex difference in tactile acuity is fully explained by the effect of finger size. This does not mean, however, that finger size is the only determinant of tactile acuity. Indeed, we note that, even accounting for finger size, much variance in tactile acuity remains unexplained (Fig. 2c). Apparently, tactile acuity is influenced by: (1) finger size (which gives rise to the sex difference), and (2) other sources, unrelated to finger size or sex.

What are these other sources of variance in acuity? Three broadly defined processing stages underlie tactile perception, and factors that affect any stage could cause individual differences in tactile spatial acuity. First, a tactile stimulus deforms the skin. Second, mechanoreceptors transform the skin deformation into an afferent population response-a peripheral neural image of the impressed sur-

anatomical and physiological work: (1) that sweat pore density is a reliable correlate of Merkel cell cluster density, and (2) that the number of Merkel clusters in a typical SA1 receptive field is independent of finger size, so that fingers with more closely spaced Merkel clusters will have more (and smaller) SA1 receptive fields per unit area (Fig. $4 b$ ).

We recognize that finger size may additionally (or exclusively) correlate with factors other than Merkel cell density to affect tactile spatial acuity. One such factor is Merkel cell depth. If fingers scale uniformly in size such that the relative spacing between components is preserved, then Merkel cells would reside more deeply within the epidermis of larger fingers. In this case, tactile thresholds would be expected to correlate positively with finger size, as we have observed, because the epidermal deformation caused by a stimulus surface diminishes with increasing depth (Phillips and Johnson, 1981b; Sripati et al., 2006).

Other factors that might correlate with finger size to influence tactile spatial acuity include skin thickness, temperature and hydration. We are unaware of studies that have investigated corre- face. Third, central neurons interpret this peripheral neural image. We have suggested that finger size, via its influence on receptor density, affects the second stage of processing. Other factors, described below, could affect the first and third stages.

Individual differences in mechanical skin properties might affect the first stage of tactile processing. Two such properties are skin compliance (Woodward, 1993) and conformance (VegaBermudez and Johnson, 2004; Gibson and Craig, 2006). A forcecontrolled stimulus will cause greater skin deformation (strain) when applied to a more compliant or conformant (pliable) finger. SA1 afferents fire at a rate proportional to the compressive (Phillips and Johnson, 1981b) or tensile (Sripati et al., 2006) strain in the epidermis at the depth of the Merkel cells. Therefore, an SAl afferent in more compliant skin will be more strongly activated by the same fixed-force stimulus. Nevertheless, the variation in skin compliance among our participants was apparently not sufficient to exert measurable effects on tactile acuity, a result consistent with that found by Woodward (1993), and perhaps not surprising in light of the insensitivity of grating orientation 
thresholds to increases in skin displacement produced by forces $>50 \mathrm{~g}$ (Gibson and Craig, 2006). Presumably, as suggested by Gibson and Craig (2006), the afferent spatial image is sufficiently clear at $50 \mathrm{~g}$ force that little or no benefit accrues to spatial perception from further increases in skin deformation. The literature on the influence of conformance is mixed, however, and deserves further investigation. Vega-Bermudez and Johnson (2004) report a correlation among young participants between a conformance-associated measure and GOT threshold, whereas Gibson and Craig (2006) report that conformance does not determine acuity on the GOT.

Individual differences in central neural circuitry could affect the third stage of tactile processing. The detailed circuitry that underlies tactile perceptual inference is unknown, but it is clear that the peripheral neural image evoked by a spatially structured surface contains more information than is typically extracted by the brain (Wheat and Goodwin, 2001; Goldreich, 2009). Because central processing is suboptimal in this sense, room for improvement exists. One salient feature of central perceptual areas, known to vary across individuals, is representational size. The representation of the fingertip in the somatosensory homunculus expands with tactile experience (Jenkins et al., 1990; PascualLeone and Torres, 1993; Sterr et al., 1999). In addition, cortical neuronal excitability increases following repetitive tactile stimulation (Höffken et al., 2007), and neuronal firing properties are modified by selective tactile attention (Roy et al., 2007). These and other central neural properties presumably account for some individual variation in tactile acuity.

In conclusion, we have shown that finger size variation accounts for the sex difference in passive tactile spatial acuity. We suggest that diminutive digits discern delicate details because within such digits Merkel cell density is high, and consequently SA1 receptive fields are closely spaced and small, resulting in excellent spatial resolution. If the influence of finger size on tactile acuity applies throughout development, then we would expect children to outperform young adults on tactile spatial tasks, as observed in one study (Stevens and Choo, 1996; but see Bleyenheuft et al., 2006). Future research will investigate the extent to which Merkel cell density and other factors-peripheral and central-influence tactile perception throughout development and adulthood.

\section{References}

Bensmaïa SJ, Craig JC, Johnson KO (2006) Temporal factors in tactile spatial acuity: evidence for RA interference in fine spatial processing. J Neurophysiol 95:1783-1791.

Bleyenheuft Y, Cols C, Arnould C, Thonnard JL (2006) Age-related changes in tactile spatial resolution from 6 to 16 years old. Somatosens Mot Res 23:83-87.

Bolton CF, Winkelmann RK, Dyck PJ (1966) A quantitative study of Meissner's corpuscles in man. Neurology 16:1-9.

Boulais N, Misery L (2007) Merkel cells. J Am Acad Dermatol 57:147-165.

Craig JC, Johnson KO (2000) The two-point threshold: not a measure of tactile spatial resolution. Curr Dir Psychol Sci 9:29-32.

Dillon YK, Haynes J, Henneberg M (2001) The relationship of the number of Meissner's corpuscles to dermatoglyphic characters and finger size. J Anat 199:577-584.

Fruhstorfer H, Abel U, Garthe CD, Knüttel A (2000) Thickness of the stratum corneum of the volar fingertips. Clin Anat 13:429-433.

Gescheider GA (1997) Psychophysics: the fundamentals, 3 Ed. Mahwah, NJ: Lawrence Erlbaum.

Gibson GO, Craig JC (2002) Relative roles of spatial and intensive cues in the discrimination of spatial tactile stimuli. Percept Psychophys 64:1095-1107.

Gibson GO, Craig JC (2006) The effect of force and conformance on tactile intensive and spatial sensitivity. Exp Brain Res 170:172-181.
Goldreich D (2009) An ideal observer for passive tactile spatial perception. Paper presented at the Conference on Computational and Systems Neuroscience, Salt Lake City, UT, February-March.

Goldreich D, Kanics IM (2003) Tactile acuity is enhanced in blindness. J Neurosci 23:3439-3445.

Goldreich D, Kanics IM (2006) Performance of blind and sighted humans on a tactile grating detection task. Percept Psychophys 68:1363-1371.

Goldreich D, Wong M, Peters RM, Kanics IM (2009) A tactile automated passive-finger stimulator (TAPS). J Vis Exp 28. pii: 1374. [Free Full Text].

Goodman SN (1999) Toward evidence-based medical statistics. 2: The Bayes factor. Ann Intern Med 130:1005-1013.

Güçlü B, Mahoney GK, Pawson LJ, Pack AK, Smith RL, Bolanowski SJ (2008) Localization of Merkel cells in the monkey skin: an anatomical model. Somatosens Mot Res 25:123-138.

Höffken O, Veit M, Knossalla F, Lissek S, Bliem B, Ragert P, Dinse HR, Tegenthoff M (2007) Sustained increase of somatosensory cortex excitability by tactile coactivation studied by paired median nerve stimulation in humans correlates with perceptual gain. J Physiol 584: 463-471.

Iggo A, Muir AR (1969) The structure and function of a slowly adapting touch corpuscle in hairy skin. J Physiol 200:763-796.

Jenkins WM, Merzenich MM, Ochs MT, Allard T, Guíc-Robles E (1990) Functional reorganization of primary somatosensory cortex in adult owl monkeys after behaviorally controlled tactile stimulation. J Neurophysiol 63:82-104

Johansson RS (1978) Tactile sensibility in the human hand: receptive field characteristics of mechanoreceptive units in the glabrous skin area J Physiol 281:101-125.

Johansson RS, Vallbo AB (1979) Tactile sensibility in the human hand: relative and absolute densities of four types of mechanoreceptive units in glabrous skin. J Physiol 286:283-300.

Johnson KO (2001) The roles and functions of cutaneous mechanoreceptors. Curr Opin Neurobiol 11:455-461.

Johnson KO, Phillips JR (1981) Tactile spatial resolution: I. Two-point discrimination, gap detection, grating resolution, and letter recognition. J Neurophysiol 46:1177-1192.

Kass RE, Raftery AE (1995) Bayes factors. J Am Stat Assoc 90:773-795.

Kontsevich LL, Tyler CW (1999) Bayesian adaptive estimation of psychometric slope and threshold. Vis Res 39:2729-2737.

Lévêque JL, Dresler J, Ribot-Ciscar E, Roll JP, Poelman C (2000) Changes in tactile spatial discrimination and cutaneous coding properties by skin hydration in the elderly. J Invest Dermatol 115:454-458.

Nolano M, Provitera V, Crisci C, Stancanelli A, Wendelschafer-Crabb G, Kennedy WR, Santoro L (2003) Quantification of myelinated endings and mechanoreceptors in human digital skin. Ann Neurol 54:197-205.

Ogawa H (1996) The Merkel cell as a possible mechanoreceptor cell. Prog Neurobiol 49:317-334.

Oldfield RC (1971) The assessment and analysis of handedness: the Edinburgh inventory. Neuropsychologia 9:97-113.

Pascual-Leone A, Torres F (1993) Plasticity of the sensorimotor cortex representation of the reading finger in braille readers. Brain 116:39-52.

Phillips JR, Johnson KO (1981a) Tactile spatial resolution. II. Neural representation of Bars, edges, and gratings in monkey primary afferents. J Neurophysiol 46:1192-1203.

Phillips JR, Johnson KO (1981b) Tactile spatial resolution. III. A continuum mechanics model of skin predicting mechanoreceptor responses to bars, edges, and gratings. J Neurophysiol 46:1204-1225.

Roy A, Steinmetz PN, Hsiao SS, Johnson KO, Niebur E (2007) Synchrony: a neural correlate of somatosensory attention. J Neurophysiol 98:1645-1661.

Schady WJ, Torebjörk HE (1983) Projected and receptive fields: a comparison of projected areas of sensations evoked by intraneural stimulation of mechanoreceptive units, and their innervation territories. Acta Physiol Scand 119:267-275.

Sivia D, Skilling J. (2006) Data analysis: a Bayesian tutorial, Ed 2. New York: Oxford UP.

Sripati AP, Bensmaia SJ, Johnson KO (2006) A continuum mechanical model of mechanoreceptive afferent responses to indented spatial patterns. J Neurophysiol 95:3852-3864. 
Sterr A, Müller M, Elbert T, Rockstroh B, Taub E (1999) Development of cortical reorganization in the somatosensory cortex of adult braille students. Electroencephalogr Clin Neurophysiol Suppl 49:292-298.

Stevens JC, Choo KK (1996) Spatial acuity of the body surface over the life span. Somatosens Mot Res 13:153-166.

Van Boven RW, Hamilton RH, Kauffman T, Keenan JP, Pascual-Leone A (2000) Tactile spatial resolution in blind braille readers. Neurology 54:2230-2236.

Vega-Bermudez F, Johnson KO (2004) Fingertip skin conformance accounts, in part, for differences in tactile spatial acuity in young subjects, but not for the decline in spatial acuity with aging. Percept Psychophys 66:60-67.
Verrillo RT, Bolanowski SJ, Checkosky CM, McGlone FP (1998) Effects of hydration on tactile sensation. Somatosens Mot Res 15:93108.

Wheat HE, Goodwin AW (2001) Tactile discrimination of edge shape: limits on spatial resolution imposed by parameters of the peripheral neural population. J Neurosci 21:7751-7763.

Woodward KL (1993) The relationship between skin compliance, age, gender, and tactile discriminative thresholds in humans. Somatosens Mot Res 10:63-67.

Yamada N, Kashima Y, Inoué T (1996) Scanning electron microscopy of the basal surface of the epidermis of human digits. Acta Anat 155:242248. 